\title{
The impact of type la supernovae on main sequence binary companions
}

\author{
R. Pakmor, F. K. Röpke, A. Weiss, and W. Hillebrandt
}

\author{
Max-Planck-Institut für Astrophysik, Karl-Schwarzschild-Str. 1, 85741 Garching, Germany \\ e-mail: rpakmor@mpa-garching.mpg.de
}

Received 24 June 2008 / Accepted 13 July 2008

\begin{abstract}
Context. The nature of type Ia supernova progenitors is still unclear. The outstanding characteristic of the single-degenerate scenario is that it contains hydrogen in the binary companion of the exploding white dwarf star, which, if mixed into the ejecta of the supernova in large amounts may lead to conflicts with the observations thus ruling out the scenario.

Aims. We investigate the effect of the impact of type Ia supernova ejecta on a main sequence companion star of the progenitor system. With a series of simulations we investigate how different parameters of this system affect the amount of hydrogen stripped from the companion by the impact.

Methods. The stellar evolution code GARSTEC is used to set up the structure of the companion stars mimicking the effect of a binary evolution phase. The impact itself is simulated with the smoothed particle hydrodynamics code GADGET2.

Results. We reproduce and confirm the results of earlier grid-based hydrodynamical simulation. Parameter studies of the progenitor system are extended to include the results of recent binary evolution studies. The more compact structure of the companion star found here significantly reduces the stripped hydrogen mass.

Conclusions. The low hydrogen masses resulting from a more realistic companion structure are consistent with current observational constraints. Therefore, the single-degenerate scenario remains a valid possibility for type Ia supernova progenitors. These new results are not a numerical effect, but the outcome of different initial conditions.
\end{abstract}

Key words. stars: supernovae: general - hydrodynamics - binaries: close

\section{Introduction}

While the progenitors for type Ib/c and type II supernovae are known, type Ia supernovae (SNe Ia) still elude an identification of their progenitor system. This is an unpleasant situation given the fact that these objects are one of the most important tools to determine cosmological parameters. By virtue of empirical calibration methods (e.g., Phillips 1993) they can be used as standardizable candles for distance measurements. This calls for an understanding of the mechanism of SNe Ia; and indeed, some progress has been made in recent years in understanding the explosion mechanism in terms of thermonuclear explosions of white dwarf (WD) stars (e.g., Reinecke et al. 2002; Gamezo et al. 2003; Röpke \& Hillebrandt 2005; Röpke \& Niemeyer 2007; Mazzali et al. 2007; Röpke et al. 2007). In order to judge potential systematic errors in SN Ia cosmology, a theoretical connection between the explosion characteristics and properties of the progenitor system would be desirable. Yet despite all efforts on both the theoretical and on the observational side, the nature of the progenitor system remains enigmatic.

The stabilization of WDs against gravity does not depend on a finite energy source such as the nuclear burning in normal stars. Due to the Fermi pressure of a degenerate electron gas, single WD stars are in principle eternally stable. Thus, some additional dynamics is required in order to reach an explosive state. The most likely possibility is a WD being part of a binary system and accreting matter from its companion.

Current progenitor models distinguish between the single degenerate and the double degenerate scenario. The former (proposed by Whelan \& Iben 1973) assumes a "normal", non-degenerate star to be the binary companion - either a main sequence (MS) star or a red giant (RG). In this case, the WD accretes mass from its MS or RG companion via Roche-lobe overflow or by winds (symbiotic systems) until it approaches the Chandrasekhar mass. The densities reached in the core of the WD are then sufficiently high to trigger nuclear reactions which finally cause a thermonuclear explosion of the star (but note that an explosion before reaching the Chandrasekhar mass may also be possible, e.g. Fink et al. 2007). The double degenerate scenario (Iben \& Tutukov 1984; Webbink 1984), on the other hand, assumes a binary system of two WDs. Due to gravitational wave emission, the system becomes unstable at some point, the WDs merge, and may eventually explode in a SN Ia. A summary of arguments in favor of and against both scenarios can be found in Livio (2000). In theoretical modeling, the single-degenerate Chandrasekhar-mass scenario has received most attention recently. By constraining the amount of fuel available in the thermonuclear explosion, it provides a natural explanation for the observed uniformity of SNe Ia.

Observationally, its hard to distinguish between the progenitor scenarios. One fundamental difference is the complete absence of hydrogen in the double degenerate scenario as it assumes a merger of two carbon/oxygen WDs. In contrast, in the typical single degenerate scenario, hydrogen is the main constituent of the companion. An exception are helium-accretors in which a more evolved companion star has lost his hydrogen envelope. Thus, the WD accretes helium instead of hydrogen and hydrogen is missing in the system. Kato \& Hachisu (2003) reported a possible detection of such an object. In the standard 
scenario, however, the companion star features a hydrogen envelope; and at least some part of it is expected to be carried away by the SN Ia ejecta impacting the companion. This, in principle, causes a problem for the single-degenerate scenario, because the astronomical classification of SNe Ia rests on the absence of hydrogen-features in the spectra of these events. The hydrogen stripped off from the companion will have rather low velocities. It may thus be detectable in nebular spectra, if abundant enough. For the single-degenerate scenario it is therefore of critical importance that the mass of stripped material is sufficiently low to be still consistent with the observations.

There have been a few attempts to search for hydrogen in nebular spectra of SN Ia. Mattila et al. (2005) studied nebular spectra of SN 2001el. From modeling them, they derived an upper limit of $0.03 M_{\odot}$ of solar abundance material at velocities lower than $1000 \mathrm{~km} \mathrm{~s}^{-1}$. Recently, Leonard (2007) studied nebular spectra of SN 2005am and SN 2005cf. Based on the same model as Mattila et al. (2005), he estimated $\lesssim 0.01 M_{\odot}$ of hydrogen material for both objects. Recently also hydrogen has been detected indirectly by Patat et al. (2007) in circumstellar material of SN 2006X.

An alternative to this approach of observationally constraining the nature of the progenitor system is to directly search for the former companion star of the single-degenerate scenario in the remnants of historical galactic SNe Ia. Such a search has been carried out in the remnant of Tycho Brahe's supernova of 1572 by Ruiz-Lapuente et al. (2004), who claimed the identification of the binary companion. The star in question is a slightly evolved solar-type star, that moves with a radial velocity of $-108 \mathrm{~km} \mathrm{~s}^{-1}$ relative to the sun. It also has an atypical large tangential velocity of about $90 \mathrm{~km} \mathrm{~s}^{-1}$. A significantly larger velocity of the star compared to neighbours is expected as a result of the disappearing binary orbit. Other stars observed in the same area with similar distances move only with average radial velocities of about -20 to $-40 \mathrm{~km} \mathrm{~s}^{-1}$, with a velocity dispersion of about $20 \mathrm{~km} \mathrm{~s}^{-1}$.

On the theory side, Marietta et al. (2000) presented twodimensional hydrodynamical simulations of the impact of $\mathrm{SNe}$ Ia on their companions. They found that $0.15 M_{\odot}$ were stripped from a Roche-lobe filling MS companion. This would rule out a MS-WD system for the SN Ia analysed by Leonard (2007), if it was representative. Recently, Meng et al. (2007) pointed out that considering the effect of the mass transfer phase on the companion star may change the result significantly. They studied the impact of SNe Ia on different companion stars analytically. In contrast to Marietta et al. (2000), who assumed the structure of single MS stars for the companion, Meng et al. (2007) evolved it through the binary evolution phase before the explosion of the WD. They found at least $0.035 M_{\odot}$ of stripped hydrogen for the companion. However, this result is only a lower limit, since they did not include mass loss by vaporization from the hot surface of the star. Thus, taken at face value, the currently available theoretical studies constitute a strong case against MS+WD progenitor systems for SNe Ia.

The aim of the study presented here is to check and update the Marietta et al. (2000) calculations with the results of recent detailed binary evolution models. Ivanova \& Taam (2004) identified possible SN Ia progenitors from a parameter study of MS+WD binary evolution. Their results are in agreement with other studies in this field (e.g. Langer et al. 2000; Han $\&$ Podsiadlowski 2004). Based on these results, we present an exploration of the effect of the impact of a SN Ia on different MS companions by 3D hydrodynamical simulations. Section 2 summarizes the codes used. Section 3 demonstrates that our approach reproduces the results of Marietta et al. (2000) and presents a resolution study. Section 4 discusses an exploration of different progenitor systems and Sect. 5 derives observational implications of our results. A summary and an outlook conclude this work in Sect. 6.

\section{Modeling approach}

Two different codes are employed in this work: one to construct the companion stars mimicking a binary evolution and the other to investigate the hydrodynamical impact of the supernova on the companion star.

To evolve the companion stars we use the stellar evolution code GARSTEC of Weiss \& Schlattl (2007). It evolves stars with a given mass and metallicity to a certain age and is used to construct a solar-type companion star similar to the "HCV" scenario of Marietta et al. (2000, see Sect. 3). The code also allows to include mass loss during the evolution but does not account for a binary evolution. In our study of a variety of progenitor models (see Sect. 4), we therefore rely on the parameters of the binary evolution study by Ivanova \& Taam (2004) to construct our companion stars. For each of the models we first set up a star that fits the parameters at the onset of the mass transfer phase. At this point, a constant mass loss rate is assumed and the stellar evolution is followed for the duration of the mass transfer period. The mass loss rate employed here corresponds to the mass loss rate of the original binary models of Ivanova \& Taam (2004) averaged over the entire mass transfer phase. After following this phase, we obtain a stellar configuration that approximates the outcome of a realistic binary evolution. It is used to study the process of the interaction with the explosion ejecta once the binary WD undergoes a SN Ia.

The impact of the supernova ejecta is simulated using the smoothed particle hydrodynamics (SPH) code GADGET2 of Springel (2005). In order to set up the companion star here, we map the one-dimensional profiles of density, internal energy, and nuclear composition of the stellar evolution calculation to a particle distribution suitable for the SPH code. The mapping is done by transforming a uniform particle distribution to the given radial density profile. Details of this procedure will be explaind in (Pakmor et al. 2008). Using GADGET2, these companion stars were relaxed in a separate step for $1.0 \times 10^{4} \mathrm{~s}$ to get rid of numerical artifacts that may have been introduced by the transformation (e.g. due to the random placement of the particles).

A supernova was added to the simulation at a distance given by the orbital period of the binary system before the explosion. The supernova was set up based on the W7 model by Nomoto et al. (1984). This one-dimensional model is well tested and provides a good fit to observations of standard SN Ia. It has a kinetic energy of $1.23 \times 10^{51} \mathrm{erg}$. At the time we add the supernova model to the simulation, it has reached already the phase of homologous expansion. The impact of the supernova ejecta and the following evolution of the ejecta and the companion star are simulated for about one hour. After this time, the companion star is already relaxing and its mass and velocity have reached constant values.

The GADGET code was so far only used for cosmological simulations, with few exceptions (e.g., Morris \& Podsiadlowski 2006, 2007). To be able to use it for a stellar astrophysics problem, a few extensions of the original implementation were necessary. The following basic setup was used in these simulations:

- the smoothing length is chosen such that a sphere of its radius encloses 80 neighboring particles; 


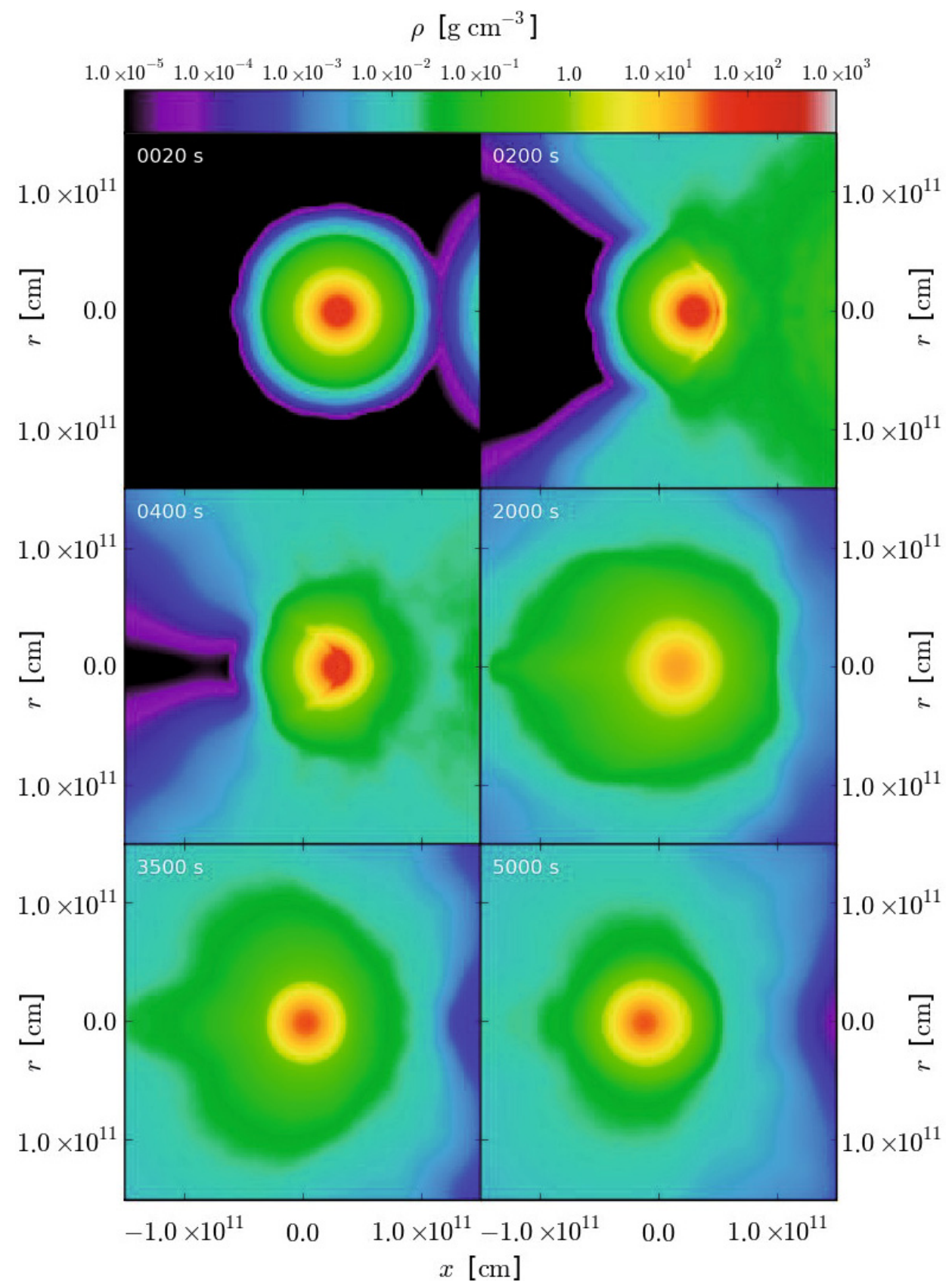

Fig. 1. Snapshots of the evolution of the companion star in the HCV scenario. The plots use cylindrical coordinates. The radial coordinate is averaged over angle. Color-coded is the density.

- the gravitational softening length equals the smoothing length;

- all particles are given the same mass;

- nuclear reactions are neglected in the simulations. This is justified since Marietta et al. (2000) showed that the additional energy generated by shock-wave induced hydrogen burning is a marginal effect. The savings in computing time due to this approximation, however, are substantial.

Details of these changes are described in a separate publication (Pakmor et al. 2008).

\section{Tests of implementation}

One of the obvious questions arising in our approach is whether the 3D SPH scheme applied here leads to the same results as the 2D grid-based approach of Marietta et al. (2000). This is tested by using the initial parameters of the HCV scenario of Marietta et al. (2000) in our setup. In the HCV scenario, the supernova is realized as a W7 model. The companion is a solar like $1.017 M_{\odot}$ main sequence star with a central hydrogen abundance of 0.58 . The separation between supernova and companion star at the time of the explosion is $2.04 \times 10^{11} \mathrm{~cm}$.

Figure 1 shows the typical evolution of the companion star in our simulations. Here, an example with a total of $235499 \mathrm{SPH}$ particles is illustrated starting out with the impact of the outermost supernova ejecta on the companion and ending when they have passed the star and it relaxes again. The first image after $20 \mathrm{~s}$ shows the companion star at the instant when the first ejecta reach the companion from the right. In the second snapshot, taken after $200 \mathrm{~s}$, the ejecta have hit the companion star. 


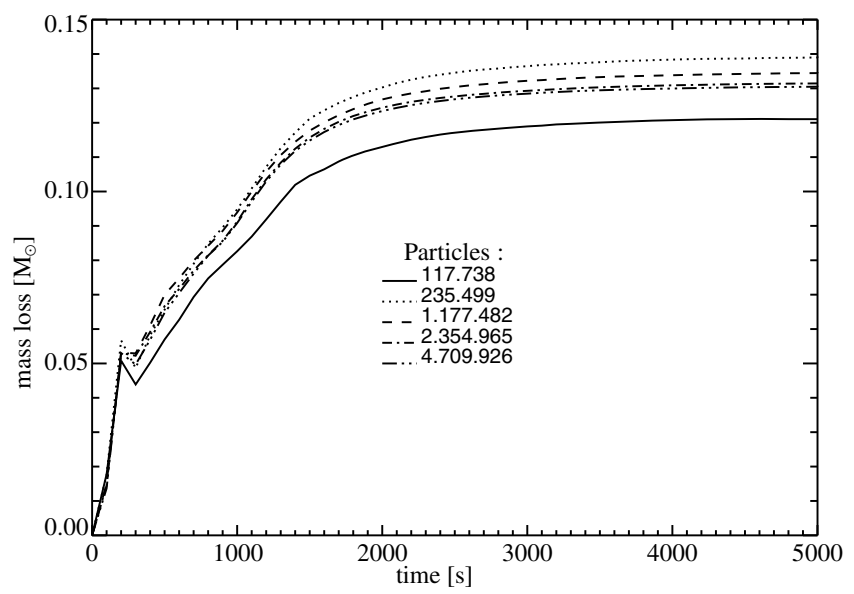

Fig. 2. Mass loss of the companion star depending on the time after the supernova explosion for different resolutions.

A shock wave forms and starts to propagate through it. Another $200 \mathrm{~s}$ later (third snapshot of Fig. 1), the shock wave reaches the center of the star. In the fourth snapshot ( 2000 s after the explosion the shock wave has crossed the star completely. Material is ejected on its far side. The last two images show the star shrinking and relaxing again.

Qualitatively, this looks similar to the simulations of Marietta et al. (2000). However, our results do not show the hydrodynamical instabilities they observed at the interface of supernova ejecta and the stripped material in the wake of the star (note that some mixing of companion star material into the supernova ejecta is realized, see Fig. 7). This difference is not surprising as SPH codes are known to suppress instabilities due to their large numerical viscosity (see, however, Fryer et al. 2007, showing that grid-based codes may under some circumstances not reproduce the mixing better than SPH codes). However, it is not a priori clear that this morphological difference significantly affects the effects and quantities we are interested in. The fundamental quantity upon which we base our comparison is the bound mass of the companion star, or, equivalently, the mass stripped away from it by the supernova ejecta.

As a first step, we perform a resolution study in order ensure a comparison with the Marietta et al. (2000) result based on a converged simulation. To test for numerical convergence we use different resolutions with $1.1 \times 10^{5}$ to $4.7 \times 10^{6} \mathrm{SPH}$ particles (counted for the entire setup, i.e. supernova and companion star, as the total mass of the configuration is equally distributed on the particles).

Figure 2 shows the evolution of the mass loss from the companion star with time for different resolutions. This value is calculated by subtracting the sum over the masses of all particles that are gravitationally bound to the star from its initial mass. Whether or not a particle is bound to the star is decided by comparing its potential energy relative to the center of the star with the kinetic energy of its motion relative to the motion of the star. For this, the center and velocity of the star are taken from the previous snapshot. Then the current center and velocity of the star are calculated from all bound particles. In principle, the new position of the star's center should be used to recalculate which particles are bound to the star and this cycle should be iterated until convergence is reached. However, already the position and velocity of the star taken from the previous snapshot usually provide a sufficiently accurate approximation, and we thus forego the iteration.
Table 1. Resolution test.

\begin{tabular}{rccccc}
\hline \hline$n_{\text {star }}$ & $n_{\text {tot }}$ & $\begin{array}{c}m_{\text {particle }} \\
{\left[M_{\odot}\right]}\end{array}$ & $\begin{array}{c}m_{\text {stripped }} \\
{\left[M_{\odot}\right]}\end{array}$ & $\begin{array}{c}m_{\text {remnant }} \\
{\left[M_{\odot}\right]}\end{array}$ & $\begin{array}{c}v_{\text {kick }} \\
{\left[\mathrm{km} \mathrm{s}^{-1}\right]}\end{array}$ \\
\hline 50000 & 117738 & $2.03 \times 10^{-5}$ & 0.126 & 0.891 & 101.1 \\
100000 & 235499 & $1.02 \times 10^{-5}$ & 0.144 & 0.873 & 95.4 \\
500000 & 1177482 & $2.03 \times 10^{-6}$ & 0.138 & 0.879 & 85.3 \\
1000000 & 2352965 & $1.02 \times 10^{-6}$ & 0.135 & 0.882 & 80.3 \\
2000000 & 4709926 & $5.09 \times 10^{-7}$ & 0.134 & 0.884 & 81.6 \\
\hline
\end{tabular}

After $3000 \mathrm{~s}$ the mass loss has stopped and the star has reached its final mass. There is a numerical artefact in the detection of the bound mass of the companion star around $\sim 300 \mathrm{~s}$ after the explosion. For one snapshot the mass of the companion star seems to increase. This is due to a false detection of particles as unbound in the previous snapshot due to high radial velocities of particles, that point to the center of the star.

Table 1 shows again some simulation properties for different resolutions after $2 \times 10^{4} \mathrm{~s}$. Here, $n_{\text {star }}, n_{\text {tot }}$, and $m_{\text {particle }}$ denote the number of particles the star is composed of, the total number of particles in the simulation, and the particle mass, respectively. The results of the simulations are characterized by mass $m_{\text {stripped }}$ stripped away from the companion star, its final mass $m_{\text {remnant }}$, and its velocity relative to the supernova $v_{\text {kick }}$.

Considering Table 1 and Fig. 2, we conclude, that this simulation is numerically converged when using more than $10^{6}$ particles. For the two simulations with 2.3 and 4.7 million particles, the graphs in Fig. 2 are nearly identical. Note that the kick velocity converges slowlier than the stripped mass.

Numerical convergence, however, does not necessarily imply consistency with the physical solution. To check our results in this respect, we compare them to previous results of Marietta et al. (2000). With the highest resolution of nearly 5 million particles the stripped mass after $2.0 \times 10^{4} \mathrm{~s}$ is $0.134 M_{\odot}$. This is quite close to the result of $0.15 M_{\odot}$ reported by Marietta et al. (2000).

As carried out here, however, the comparison is not yet based on exactly the same assumptions. Marietta et al. (2000) performed their simulations on a finite computational grid and therefore mass was lost over the domain boundaries. This mass was always assumed to be unbound from the companion star. We therefore recalculate the stripped mass in our simulations by assuming for comparison all particles to be unbound that are outside a cylindrical box equal to the simulation volume of Marietta et al. (2000). In this approach we find a stripped mass of $0.143 M_{\odot}$ for our highest resolved simulation. This result is in excellent agreement with the $0.15 M_{\odot}$ of stripped mass found by Marietta et al. (2000). Moreover, the remnant star velocities at this time $\left(85.7 \mathrm{~km} \mathrm{~s}^{-1}\right.$ reported by Marietta et al. 2000, vs. our $81.4 \mathrm{~km} \mathrm{~s}^{-1}$ ) agree very well.

We therefore conclude that our SPH approach is capable of capturing the main dynamical effects of the supernova impact on the companion star. The global quantities of the Marietta et al. (2000) study are reproduced down to the percent level. The differences in the occurrence of hydrodynamic instabilities are obviously a minor effect with respect to the overall results such as the stripped mass and the velocity of the companion star caused by the kick by the supernova ejecta. The instabilities suppressed in our SPH approach may, however, enhance the mixing between supernova ejecta and stripped material from the companion in reality. 
Table 2. Parameters of the progenitor models.

\begin{tabular}{lccccccc}
\hline \hline Model & $\begin{array}{c}M_{\mathrm{c}, \mathrm{i}} \\
{\left[M_{\odot}\right]}\end{array}$ & $\begin{array}{c}M_{\mathrm{c}, \mathrm{f}} \\
{\left[M_{\odot}\right]}\end{array}$ & $\begin{array}{c}\Delta t_{\mathrm{tr}} \\
{[\mathrm{yr}]}\end{array}$ & $\begin{array}{c}P_{\mathrm{f}} \\
{[\mathrm{d}]}\end{array}$ & $\begin{array}{c}a_{\mathrm{f}} \\
{\left[10^{11} \mathrm{~cm}\right]}\end{array}$ & $\begin{array}{c}M_{\text {stripped }} \\
\left(M_{\odot}\right)\end{array}$ & $\begin{array}{c}v_{\text {kick }} \\
{\left[\mathrm{km} \mathrm{s}^{-1}\right]}\end{array}$ \\
\hline rp3_28a & 2.8 & 0.6 & $7.7 \times 10^{5}$ & 1.7 & 5.21 & 0.032 & 52.8 \\
rp3_20a & 2.0 & 1.17 & $3.9 \times 10^{6}$ & 0.55 & 2.68 & 0.032 & 46.6 \\
rp3_20b & 2.0 & 1.25 & $2.0 \times 10^{6}$ & 1.08 & 4.26 & 0.0095 & 24.1 \\
rp3_25a & 2.5 & 1.37 & $1.7 \times 10^{6}$ & 0.51 & 2.62 & 0.058 & 60.5 \\
rp3_24a & 2.4 & 1.4 & $8.4 \times 10^{5}$ & 1.1 & 4.39 & 0.010 & 26.6 \\
rp3_20c & 2.0 & 1.46 & $2.6 \times 10^{6}$ & 1.44 & 5.29 & 0.012 & 17.0 \\
\hline
\end{tabular}

\section{Parameter studies}

Three major physical parameters of the progenitor system are expected to influence the dynamics of the supernova impact on the companion star: the kinetic energy of the supernova ejecta (powered by the thermonuclear burning in the explosion), the separation between supernova and companion, and structure of the companion star at the time of the explosion.

In reality, of course, separation and companion structure are not independent, but result from the characteristics of the original binary system and its evolution through the mass transfer phase. Moreover, the dependence of the supernova explosion energy on other parameters is unknown and thus treated as an independent physical parameter in this study. In any system with Roch-lobe overflow, the solid angle under which the companion star is seen from the white dwarf depends only on the mass ratio of its components. However, in order to determine how changing the distance affects the results it is treated here as an independent parameter. This is motivated partially by the possibility that the WD and the companion star disconnect shortly before the explosion, as suggested by Patat et al. (2007) for SN 2006X.

Our main emphasis, however, will be on the structure of the companion star. In order to model the progenitor systems, we take parameters from the study of Ivanova \& Taam (2004) and a typical Population I metallicity of 0.02 for all stars. Ivanova \& Taam (2004) analyzed the evolution of possible SN Ia progenitor systems consisting of a WD and an evolved MS star. Detailed calculations of 65 different systems were carried out, varying the initial mass of both objects and the initial distance to cover the parameter space. From the resulting sample, they pick six representative models which are believed to be likely to evolve into a SN Ia. These are the systems we select for our study. Their properties are listed in Table 2 . In addition, characteristics of the companion star after interaction with the supernova ejecta are given.

Values taken from Ivanova $\mathcal{E}$ Taam (2004): masses of the companion star at the beginning of the mass transfer $M_{\mathrm{d}, \mathrm{i}}$ and at the time of the explosion $M_{\mathrm{d}, \mathrm{f}}$, length of the mass transfer period $\Delta t_{\mathrm{tr}}$, orbital period $P_{\mathrm{f}}$, distance between white dwarf and its companion star just before the explosion $a_{\mathrm{f}}$.

Results of the simulations: mass stripped from the companion $M_{\text {stripped }}$ and its kick velocity $v_{\text {kick }}, 5000 \mathrm{~s}$ after the explosion.

The three parameters of the progenitor system are individually discussed in the following paragraphs. While the simulations testing the effects of the explosion energy and the binary separation were carried out with $2 \times 10^{5}$ particles in the companion star, the study based on the realistic progenitor system structure was set up with $2 \times 10^{6}$ and $4 \times 10^{6}$ particles representing the companion. Therewith, the mass of the single particles and, as all particles had the same mass by construction, the number of particles representing the supernova explosion was fixed.

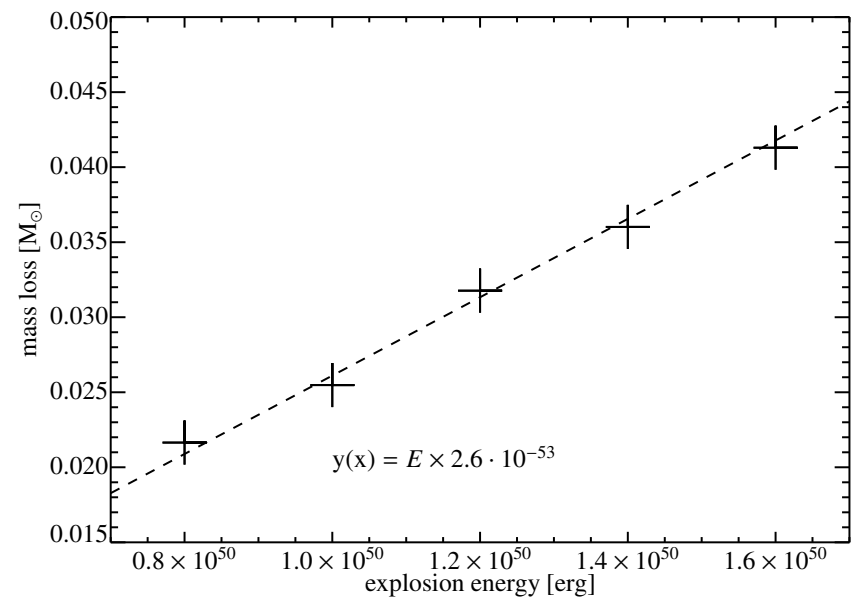

Fig. 3. Stripped mass for different supernova energies in model rp3_20a.

\subsection{Explosion energy}

The influence of the supernova explosion energy on the interaction with the companion is studied on the basis of model rp3_20a. All parameters but the supernova energy were kept constant with the values of the original model (see Table 2).

The kinetic energy of the supernova was varied in the range $0.8 \ldots 1.6 \mathrm{~B}\left(=0.8 \ldots 1.6 \times 10^{51} \mathrm{erg}\right)$. The lower limit corresponds to lowest kinetic energies of simulated deflagration SNe Ia. The upper limit is the maximum energy a SN Ia can have, assuming that a Chandrasekhar-mass WD consisting of an equal-by-mass mixture of $\mathrm{C}$ and $\mathrm{O}$ burns completely to $\mathrm{Ni}^{56}$. The kinetic energy of the supernova ejecta $E_{\mathrm{kin}, \mathrm{SN}}^{\prime}$ was adjusted by scaling the velocities $\boldsymbol{v}^{\prime}$ of the supernova particles (originally representing the W7 model with $E_{\mathrm{kin}, \mathrm{SN}}^{\mathrm{W} 7}$ and $\boldsymbol{v}^{\mathrm{W} 7}$ ) according to

$\boldsymbol{v}^{\prime}=\sqrt{\frac{E_{\mathrm{kin}, \mathrm{SN}}^{\prime}}{E_{\mathrm{kin}, \mathrm{SN}}^{\mathrm{W} 7}}} \cdot \boldsymbol{v}^{\mathrm{W} 7}$.

This scaling preserves the homologous expansion $(v \propto r)$ of the ejecta.

The stripped mass as a function of the supernova energy is shown in Fig. 3. The relation is linear in good approximation and can be fitted by

$M_{\text {stripped }}=2.6 \times 10^{-2} \frac{E_{\mathrm{kin}, \mathrm{SN}}}{10^{51} \mathrm{erg}} M_{\odot}$

assuming that the offset is zero (without this constraint the offset is only $1.3 \times 10^{-3}$ ). We emphasize that, although the functional form of the relation may be generic, the particular parameters of the fit apply to model rp3_20a only. For different companion structures, for instance, the values of the parameters are expected 
to change. This should be kept in mind for the fits presented below as well.

Note that the studied energy range covers only a factor of 2 and therefore the stripped mass also changes only by a factor of 2 . The energy of the W7 model of $1.23 \times 10^{51} \mathrm{erg}$ corresponds to an intermediate case. These results indicate that changes in the supernova energy have only a small effect on the stripped mass as compared to the other parameters discussed below. The kick velocity can be fitted by a power law as

$v_{\text {kick }}=2.5 \times 10^{-22}\left(\frac{E_{\text {kin }, \mathrm{SN}}}{\mathrm{erg}}\right)^{0.55} \mathrm{~cm} \mathrm{~s}^{-1}$.

A simple argument for this behavior may be that velocity of the companion is mainly given by the momentum exchange from the supernova ejecta that hit the star. As the velocity of the ejecta increases with the square root of the kinetic energy, the velocity of the star should increase accordingly. The exponent in Eq. (3) is only slightly larger than $1 / 2$ and thus this simple picture captures the process rather well.

\subsection{Distance}

In order to test the influence of the separation, we again choose model rp3_20a (see Table 2 for the parameters). Here, the distance $d$ between WD and companion was varied in the range of $1.5 \ldots 3 \times 10^{11} \mathrm{~cm}$. All other parameters were kept constant. The relation between distance and stripped mass for this model is shown in Fig. 4. From the largest to the smallest distance the stripped mass increases by a factor of 10 . The relation follows a power law in good approximation and can be fitted to

$M_{\text {stripped }}=2.38 \times 10^{38}\left(\frac{d}{\mathrm{~cm}}\right)^{-3.49} M_{\odot}$.

The kick velocity depending on the separation $d$ also follows a power law. It is approximately given by

$v_{\text {kick }}=2.5 \times 10^{23}\left(\frac{d}{\mathrm{~cm}}\right)^{-1.45} \mathrm{~cm} \mathrm{~s}^{-1}$.

The fraction of the supernova ejecta that hits the star scales with the inverse square of the distance treated as an independent parameter as discussed above. Remembering that the stripped mass seems to scale linearly with the explosion energy, one may expect a geometrical scaling as $M_{\text {stripped }} \propto d^{-2}$. This does clearly not fit our results. It indicates that the connection is more complex, and the linear correlation between explosion energy and stripped mass is possibly only a result of the small energy range tested. In addition the simple scaling neglects that the companion star is not flat disc but a sphere. The whole process also seems to depend on the time evolution of the density of the ejecta hitting the star.

\subsection{Companion star}

Table 2 shows the stripped masses and the kick velocities of the remnants for the six different progenitor systems suggested by Ivanova \& Taam (2004). The supernova model used in all models is the original W7 model with a total kinetic energy of $1.23 \times$ $10^{51} \mathrm{erg}$. The variations in the stripped mass between the $2 \times 10^{6}$ particles and the $4 \times 10^{6}$ particles simulations were less than a few percent indicating numerically converged results.

The stripped masses range from $0.01 M_{\odot}$ to $0.06 M_{\odot}$ for the different setups. This is significantly less than previous results

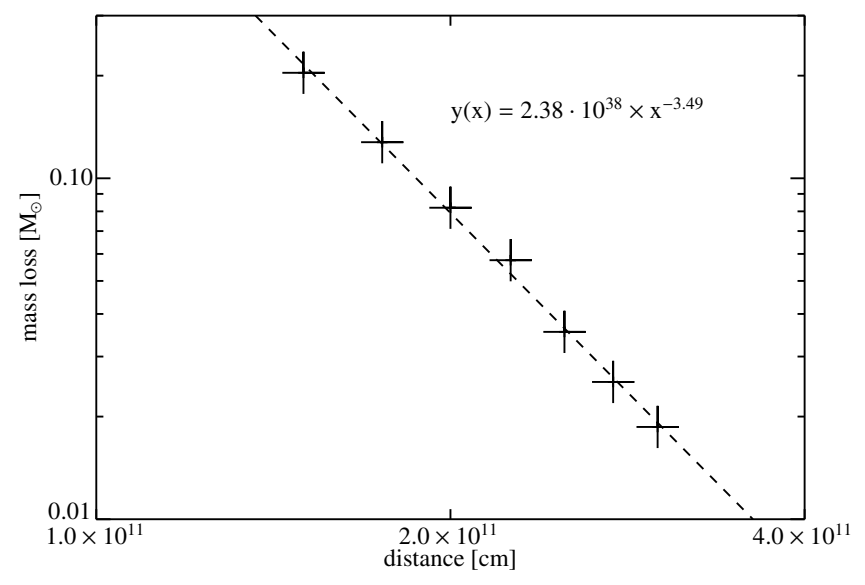

Fig. 4. Stripped mass versus binary separation for model rp3_20a.

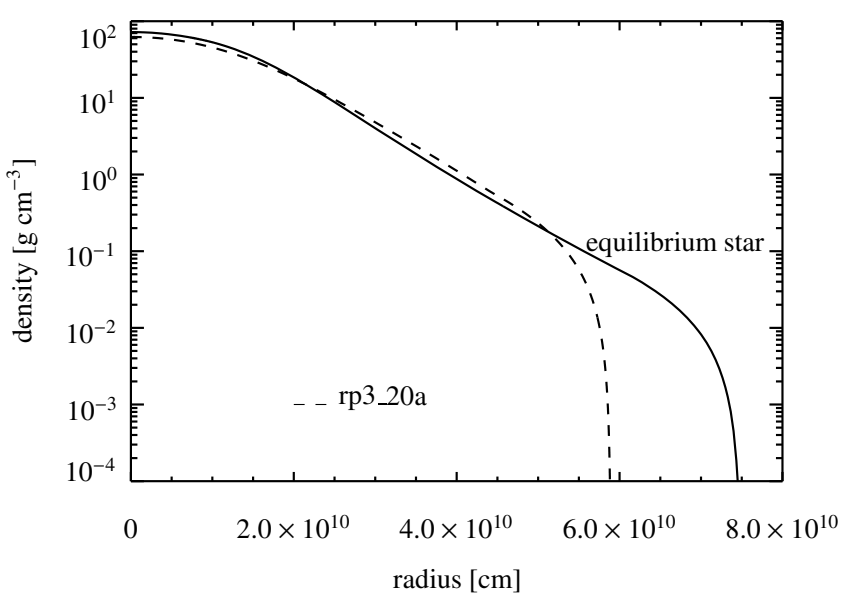

Fig. 5. Comparison of density profiles of the companion star in model rp3_20a at the time of the explosion and a single star with the same mass and nuclear age.

reported by other authors (Marietta et al. 2000; Meng et al. 2007). Compared to the $0.15 M_{\odot}$ result of Marietta et al. (2000) we find a factor of 3-15 less stripped material.

This deviation is attributed to the binary evolution of the progenitor we take into account in the present study. The binary evolution significantly affects the properties of both the companion star and the geometry of the binary configuration.

The main effect on the companion star is illustrated in Fig. 5, where the density profile of the companion star in model rp3_20a at the time of the explosion is plotted in comparison with the density profile of a star in thermal equilibrium that was evolved as a single object to the same mass and nuclear age. The equilibrium star features a much larger radius than the binary companion. This is a result of the mass transfer phase in the binary system, that removes the outer layers and leads to a more compact star. The mass loss occurs rather fast: the duration of the mass transfer phase is about a factor of 10 less than the KelvinHelmholtz time-scale of the stars in our models. Therefore, the star is not able to adjust its structure to the loss of the outer layers and it shrinks into a more compact object. Finding less stripped mass when taking into account the modification of the companion structure due to the binary evolution is thus not surprising.

In order to corroborate this interpretation, we set up model rp3_20a with a companion structure that corresponds to that of a star of equal mass in thermal equilibrium. In this model, $0.066 M_{\odot}$ are stripped from the companion by the supernova. 


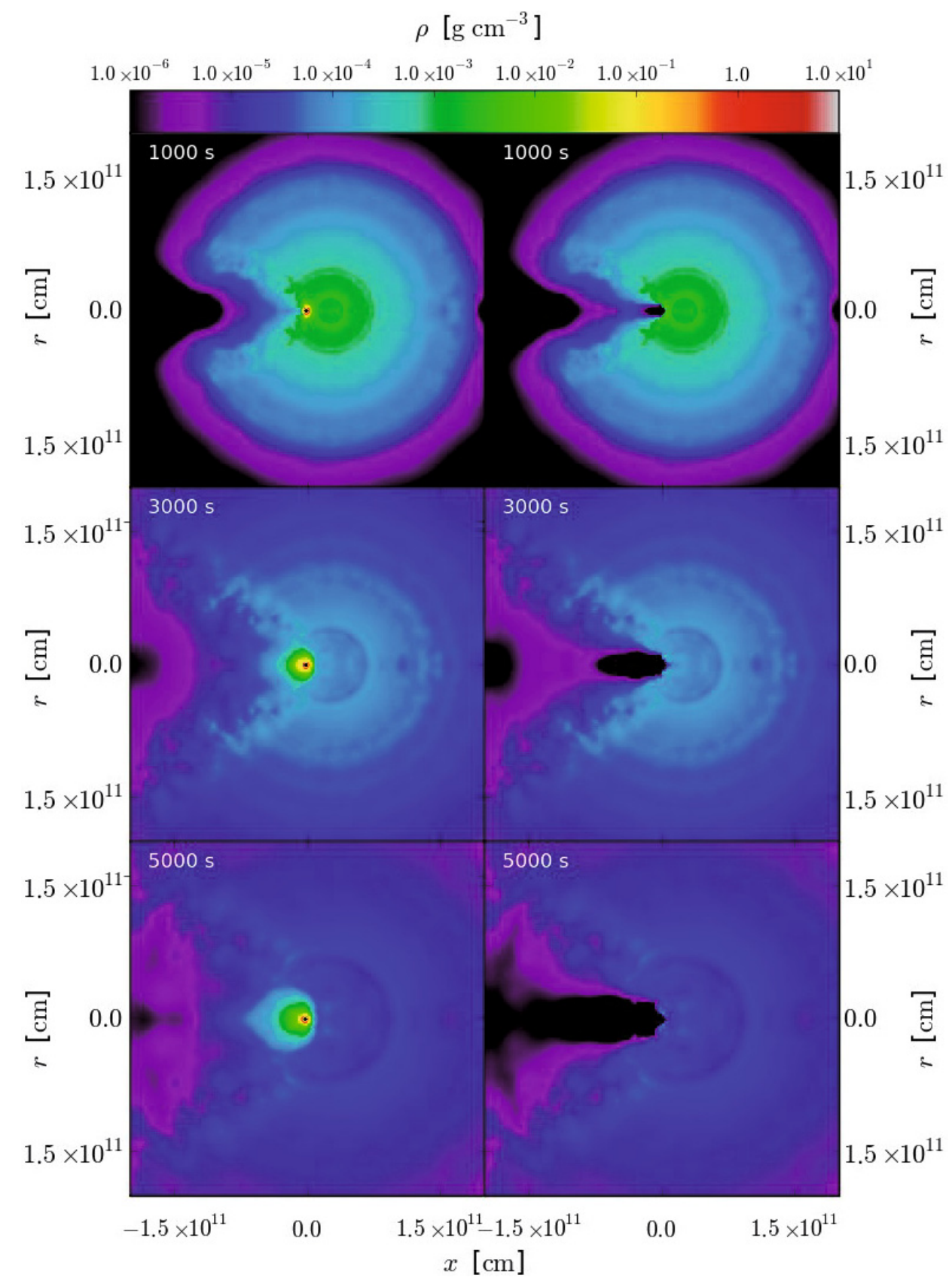

Fig. 6. Snapshots of the evolution of the supernova ejecta in the rp3_20a scenario. The left column shows supernova ejecta and the companion star. In the right column all material of the companion is cut out, leaving only the supernova material. The plots use cylindrical coordinates. The radial coordinate is averaged over angle. Color-coded is the density.

This is a factor of 2 more than for the original rp3 20a model. The difference can be explained by the larger, less bound envelope of the equilibrium star that can be stripped away more easily. Moreover, the larger radius causes an extended interaction area that also leads to a slightly larger kick velocity of $51.2 \mathrm{~km} \mathrm{~s}^{-1}$ for the equilibrium star companion model evolved in isolation as compared to the $46.6 \mathrm{~km} \mathrm{~s}^{-1}$ of the binary star companion model.

Additionally, in the model we use the companion star is slightly more massive $\left(1.17 M_{\odot}\right.$ at the time of the explosion) than the solar mass companion star of Marietta et al. (2000). This leads to a slightly larger radius of the equilibrium star companion and therewith a larger separation distance and a decreased stripped mass.
These effects together explain the significant difference between our results and the previous work of Marietta et al. (2000), who used a companion with the structure of an isolated equilibrium star. We note, however, that our interpretation is not in agreement with the work of Meng et al. (2007), who found a lower limit on the stripped mass of $0.035 M_{\odot}$ taking into account binary evolution. This is probably be due to oversimplifying assumptions they made in their analytical treatment of the ejectacompanion star interaction.

The kick velocities of the companion star after the impact of the supernova ejecta we find in our models vary from $17 \mathrm{~km} \mathrm{~s}^{-1}$ to $61 \mathrm{~km} \mathrm{~s}^{-1}$ (see Table 2). The velocities roughly increase with the size of the companion stars (as the cross section increases) and decrease with larger separation distances (as the transferred 


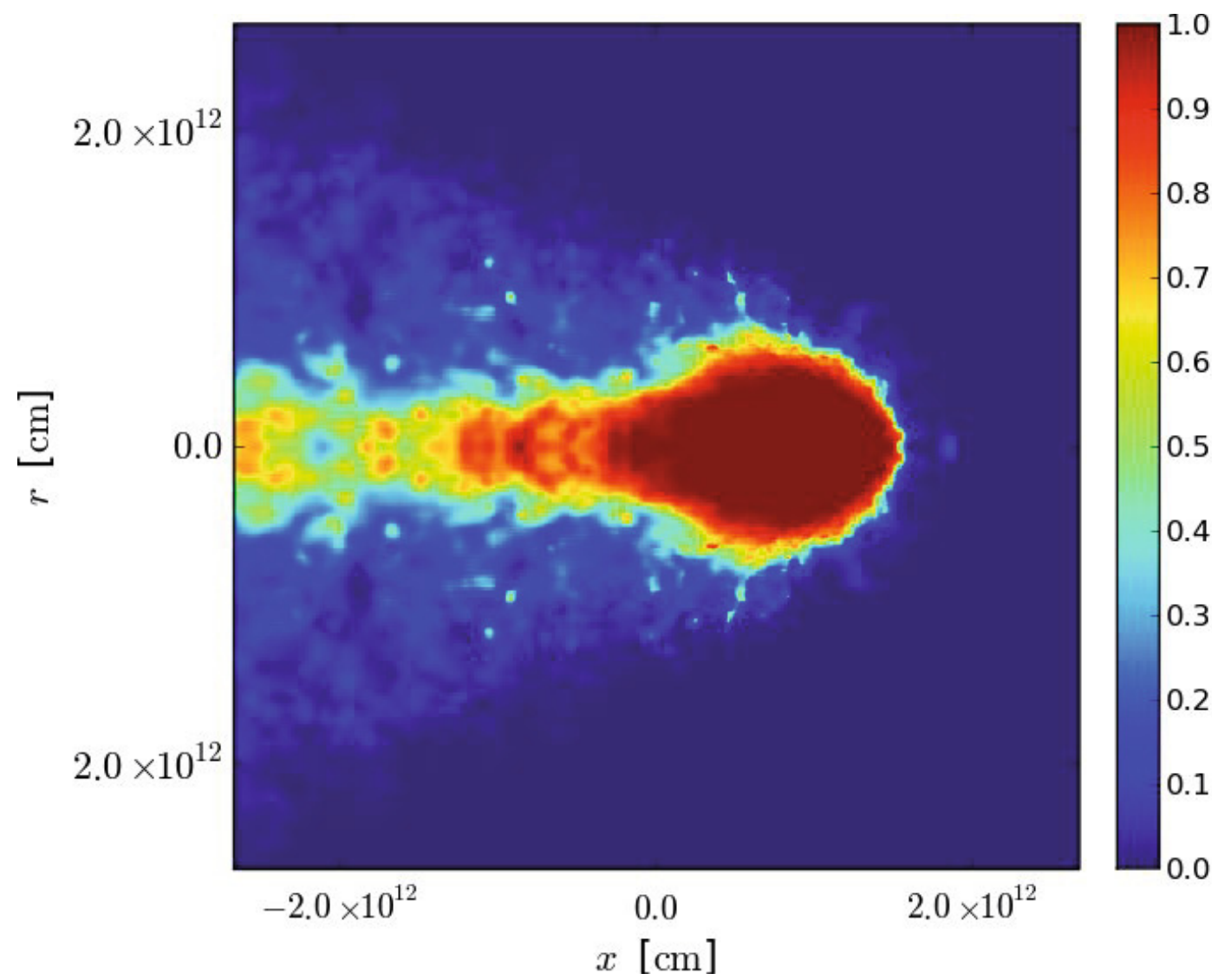

Fig. 7. Snapshot of simulation rp3_20a taken $5000 \mathrm{~s}$ after the explosion. Color-coded is the of the relative amount of material originally belonging to the companion star (red) with respect to the total material (blue corresponds to supernova material). Cylindrical coordinates with the radial coordinate averaged over angle are used.

momentum decreases) and masses (for the same transferred momentum) of the companion stars.

\section{Observational implications}

Observations that may help to constrain the nature of the SN Ia progenitor systems are the detection of hydrogen in the spectra of these events, signatures in the spectrapolarimetry data due to an asymmetric morphology of the ejecta as a result of the interaction with the companion star, and a direct observation of companions in supernova remnants. We discuss these possibilities in the light of the presented study.

\subsection{Hydrogen detection in SN la spectra}

Our results are consistent with the constraints on hydrogen in the ejecta as given by Leonard (2007) and Mattila et al. (2005). However, none of our models has stripped hydrogen mass far below the upper limit of Leonard (2007) of $0.01 M_{\odot}$. As these limits result from a non-detection of hydrogen in nebular spectra, our simulations predict hydrogen detection not far below these values. Conclusions on the validity of the single-degenerate scenario depend on whether the systems studied here are representative for Chandrasekhar mass WD+MS SN Ia progenitors and whether the observed events fall into this class. If both were true, lowering the observational upper limits of hydrogen in the ejecta of SNe Ia by another order of magnitude would exclude this progenitor scenario.
Yet it is important to note that a quite simple model was employed by Leonard (2007) and Mattila et al. (2005) to constrain limits on the hydrogen mass from the observations. A more rigorous approach would be to use the results of hydrodynamical simulations such as presented here as an input for full radiative transport calculations. From these calculations it will be possible to predict whether hydrogen lines should be visible in the spectra and how strong they should be at a given epoch. This issue will be addressed in a forthcoming study.

\subsection{Hole in the ejecta}

The supernova ejecta do not only affect the companion star, but are also affected themselves by the impact. Figure 6 shows the material after the impact. The left column shows both the supernova ejecta and the companion star material including the hydrogen stripped from the star. The right column shows only the material that was part of the supernova ejecta at the beginning of the simulation.

The supernova ejecta that were spherically symmetric in the beginning are clearly asymmetric after the impact. In the wake of the companion star, a cone-like hole in the supernova ejecta is visible. To some degree it is filled with material that is ejected from the companion star. At the borders of this hole, the supernova ejecta are slightly denser, because the material missing in the hole was transfered there. The opening angle of the conelike hole is about $45^{\circ}$ (see the top row of Fig. 6 showing the ejecta $1000 \mathrm{~s}$ after the explosion). This result is consistent with the findings of Marietta et al. (2000). However, the area in which 
the supernova ejecta are affected by the impact on the companion star is as large as $90^{\circ}$ (see middle and the lower row of Fig. 6 showing the density of supernova ejecta after $3000 \mathrm{~s}$ and $5000 \mathrm{~s}$ ).

A more detailed view of how the supernova ejecta are mixed with the material stripped from the companion star is given in Fig. 7. It shows for the the same setup the relative amount of material that originally belonged to the companion star with respect to the total amount of material.

Qualitatively, the structure of the ejecta resembles the simple model used by Kasen et al. (2004) to explore the effect of a hole in the ejecta on spectra and luminosity of SN Ia. With it he was able to reproduce observed spectrapolarimetry observations of SN Ia.

\subsection{Identifying companion stars in supernova remnants}

The remaining companion star should have a different velocity than its surrounding stars. This velocity is determined by the orbital velocity of the star at the moment the white dwarf explodes and of the kick it gets from the impact of the supernova ejecta. The former is perpendicular to the connecting line between the white dwarf and the companion star. For our models it ranges between $130 \mathrm{~km} \mathrm{~s}^{-1}$ (rp3_20c) and $380 \mathrm{~km} \mathrm{~s}^{-1}$ (rp3_28a). The latter velocity is a result of the impact of the ejecta and is therefore aligned with the connecting line. In our simulations it reaches values from $17 \mathrm{~km} \mathrm{~s}^{-1}$ to $50 \mathrm{~km} \mathrm{~s}^{-1}$. Therefore, the orbital velocity clearly dominates the velocity of the companion star relative to the center of the supernova remnants. The velocity of the star Tycho $\mathrm{G}$ which was identified as progenitor of Tycho Brahe's supernova by Ruiz-Lapuente et al. (2004) features a modulus of its spatial velocity of $136 \mathrm{~km} \mathrm{~s}^{-1}$. It thus falls into lowest part of the range we find in our simulations. The properties of Tycho G are thus consistent with the predictions of our models.

\section{Conclusions}

We studied the impact of the ejecta of $\mathrm{SNe}$ Ia on main sequence companion stars in the context of the single-degenerate Chandrasekhar-mass scenario with hydrodynamical simulations. To this end, the (cosmological) GADGET2 smoothed particle hydrodynamics code was adapted to the stellar problem and employed in numerical simulations. It was shown that this SPHbased approach is capable of reproducing previous results obtained with a grid-based 2D scheme by Marietta et al. (2000). A resolution study indicated that with a few million particles the simulations yield numerically converged results.

We showed that the mass stripped from the companion star by the impact of the ejecta depends on the their kinetic energy and the binary separation. While the latter affects the mass of the stripped material significantly, the former has only a minor effect on it. This is due to the fact that the supernova explosion energy can only vary in a relatively narrow range given the restricted amount of fuel available for the nuclear energy generation.

The SPH approach was used to analyze the impact in a number of more realistic progenitor models than those employed in previous studies. For these, the companion stars were constructed with the stellar evolution code GARSTEC mimicking binary mass transfers with the parameters given by Ivanova \& Taam (2004). In the hydrodynamical impact simulations, we found about one order of magnitude less hydrogen material stripped off the companion by the impact of the supernova than predicted by previous studies. The main reason for this difference is a modified, more compact stellar structure of the companion star in combination with a resulting variation in the separation distance of the progenitor system. In particular the more compact state of the companion impedes the mass loss in the impact.

The reduced amount of hydrogen mixed into the ejecta of the supernova as predicted by our simulations leads to an agreement with observational studies of SN Ia nebular spectra (Mattila et al. 2005; Leonard 2007). This removes the former disagreement between the available observations and simulations of the $\mathrm{WD}+\mathrm{MS}$ progenitor system. Thus, to current knowledge, such a progenitor scenario is admissible in the context discussed here.

However, since the hydrogen masses predicted by our simulations are not far below the current observational upper limits, it may be possible in the near future to confirm or reject the studied progenitor scenario by either detecting hydrogen in SNe Ia or lowering the limits by another order of magnitude. A stringent way of analysis would be to calculate synthetic spectra directly from the presented simulations and to compare the results with observations. This will be tackled in a forthcoming study.

Acknowledgements. We thank Hans Ritter for helpful discussions on the binary evolution of the progenitor systems. Volker Springel and Klaus Dolag gave invaluable support with the numerical implementation into the framework of the the GADGET2 code. The research of F.K.R. is supported through the Emmy Noether Program of the German Research Foundation (DFG; RO 3676/1-1). Additional support came from the Cluster of Excellence EXC 153 "Origin and Structure of the Universe" and from the Transregional Collaborative Research Center TR 33.

\section{References}

Fink, M., Hillebrandt, W., \& Röpke, F. K. 2007, A\&A, 476, 1133

Fryer, C. L., Hungerford, A. L., \& Rockefeller, G. 2007, Inter. J. Mod. Phys. D, 16,941

Gamezo, V. N., Khokhlov, A. M., Oran, E. S., Chtchelkanova, A. Y., \& Rosenberg, R. O. 2003, Science, 299, 77

Han, Z., \& Podsiadlowski, P. 2004, MNRAS, 350, 1301

Iben, Jr., I., \& Tutukov, A. V. 1984, ApJS, 54, 335

Ivanova, N., \& Taam, R. E. 2004, ApJ, 601, 1058

Kasen, D., Nugent, P., Thomas, R. C., \& Wang, L. 2004, ApJ, 610, 876

Kato, M., \& Hachisu, I. 2003, ApJ, 598, L107

Langer, N., Deutschmann, A., Wellstein, S., \& Höflich, P. 2000, A\&A, 362, 1046 Leonard, D. C. 2007, ApJ, 670, 1275

Livio, M. 2000, in type Ia Supernovae, Theory and Cosmology, ed. J. C. Niemeyer, \& J. W. Truran (Cambridge University Press), 33

Marietta, E., Burrows, A., \& Fryxell, B. 2000, ApJS, 128, 615

Mattila, S., Lundqvist, P., Sollerman, J., et al. 2005, A\&A, 443, 649

Mazzali, P. A., Röpke, F. K., Benetti, S., \& Hillebrandt, W. 2007, Science, 315, 825

Meng, X., Chen, X., \& Han, Z. 2007, PASJ, 59, 835

Morris, T., \& Podsiadlowski, P. 2006, MNRAS, 365, 2

Morris, T., \& Podsiadlowski, P. 2007, Science, 315, 1103

Nomoto, K., Thielemann, F.-K., \& Yokoi, K. 1984, ApJ, 286, 644

Pakmor, R., Röpke, F. K., Springel, V., \& Hillebrandt, W. 2008, in preparation

Patat, F., Chandra, P., Chevalier, R., et al. 2007, Science, 317, 924

Phillips, M. M. 1993, ApJ, 413, L105

Reinecke, M., Hillebrandt, W., \& Niemeyer, J. C. 2002, A\&A, 391, 1167

Röpke, F. K., \& Hillebrandt, W. 2005, A\&A, 431, 635

Röpke, F. K., \& Niemeyer, J. C. 2007, A\&A, 464, 683

Röpke, F. K., Hillebrandt, W., Schmidt, W., et al. 2007, ApJ, 668, 1132

Ruiz-Lapuente, P., Comeron, F., Méndez, J., et al. 2004, Nature, 431, 1069

Springel, V. 2005, MNRAS, 364, 1105

Webbink, R. F. 1984, ApJ, 277, 355

Weiss, A., \& Schlattl, H. 2007, Ap\&SS, 341

Whelan, J., \& Iben, I. J. 1973, ApJ, 186, 1007 